
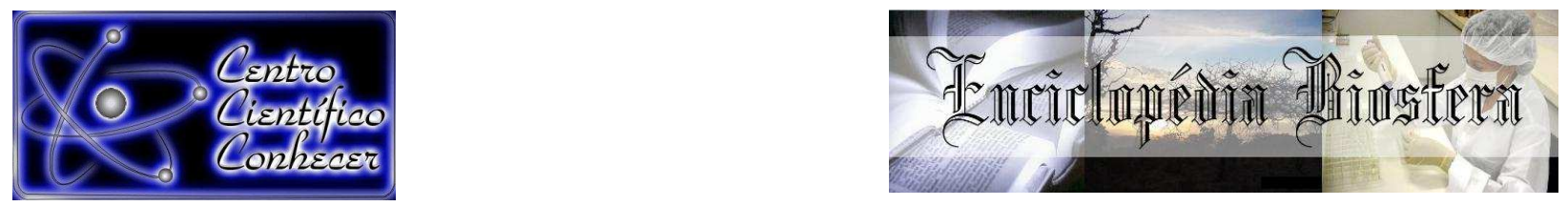

\title{
INFLUÊNCIA DA MASSA DE CATALISADOR NOS CUSTOS E RECEITAS DE UM REATOR CATALÍTICO DE LEITO FIXO
}

\author{
Alexandre Martins Ribeiro ${ }^{1}$, Roniérik Pioli Vieira ${ }^{2}$ \\ ${ }^{1}$ Graduando em Engenharia Química no Instituto Federal de Educação, Ciência e \\ Tecnologia do Sul de Minas Gerais - IFSULDEMINAS, Campus Pouso Alegre - \\ Brasil. (alexandremribeiro96@hotmail.com) \\ ${ }^{2}$ Professor Doutor do curso de Engenharia Química do Instituto Federal de \\ Educação, Ciência e Tecnologia do Sul de Minas Gerais - IFSULDEMINAS, Campus \\ Pouso Alegre - Brasil.
}

Recebido em: 02/10/2017 - Aprovado em: 21/11/2017 - Publicado em: 05/12/2017 DOI: 10.18677/EnciBio 2017B125

\begin{abstract}
RESUMO
Este trabalho consiste em uma análise da influência da massa de catalisador nos custos e receitas de um reator catalítico de leito fixo (PBR), que pode ser empregada para fins didáticos em cursos de graduação em engenharia química. Desenvolveuse um modelo matemático para o PBR considerando um sistema genérico em fase gasosa, com perda de carga no leito. Em seguida, dispondo do sistema de equações e de seus parâmetros, uma simulação forneceu a influência da concentração de um dado reagente " $\mathrm{B}$ " em excesso sobre o lucro obtido na venda de um produto " $\mathrm{C}$ ". Obteve-se um lucro de $115 \%$ em relação à massa de catalisador adquirida $(10 \mathrm{~kg})$ para $\mathrm{C}_{\mathrm{B} 0}=0,4 \mathrm{M}$; enquanto que para $\mathrm{C}_{\mathrm{B} 0}=0,2 \mathrm{M}$ houve um prejuízo de $17 \%$ para a mesma massa de catalisador utilizada no primeiro mês de operação do sistema. A análise foi proposta de forma simples, sendo assim, o problema proposto pode ser uma nova ferramenta para complementar o ensino de engenharia química.
\end{abstract}

PALAVRAS-CHAVE: avaliação econômica, PBR, reator de leito fixo.

\section{INFLUENCE OF CATALYST MASS ON THE COSTS AND INCOMES OF A CATALYTIC PACKED BED REACTOR}

\begin{abstract}
This work presents an analysis of catalyst mass influence on the costs and incomes of a catalytic PBR, which can be used for didactic purposes in chemical engineering undergraduate courses. A PBR mathematical modeling was developed considering a generic system, in the gas phase, with pressure drop. Then, having the equations system and their parameters, simulation provided the influence of a given reagent "B" concentration in excess on the profit by selling " $\mathrm{C}$ ". A gain of $115 \%$ was obtained with respect to the mass of catalyst purchased $(10 \mathrm{~kg})$ for $\mathrm{C}_{\mathrm{B} 0}=0.4 \mathrm{M}$; While for $\mathrm{C}_{\mathrm{B} 0}=0.2$ $\mathrm{M}$, there was a $17 \%$ loss for the same mass of catalyst used in the first month of system operation. The analysis was proposed in a simple way, so the proposed problem could be a new tool to complement the teaching of chemical engineering.
\end{abstract}

KEYWORDS: economic analysis, fixed bed reactor, PBR.

ENCICLOPÉDIA BIOSFERA, Centro Científico Conhecer - Goiânia, v.14 n.26; p.1498 2017 


\section{INTRODUÇÃO}

A modelagem e simulação de processos, em conjunto, constituem uma ferramenta de grande importância para os engenheiros químicos. Por exemplo, nas fases iniciais do projeto, tal ferramenta contribui para a análise e dimensionamento do sistema, bem como na identificação de seus parâmetros chave (VIEIRA; LONA, 2015). Por outro lado, uma vez que um processo está consolidado, a modelagem e simulação podem fornecer informações sobre as melhores condições de operação, viabilizando o trabalho em um sistema otimizado (CHACHUAT; LATIFI, 2003; SILVA et al., 2016; VIEIRA; LONA, 2016).

Nas disciplinas de cálculo de reatores, ministradas nos cursos de engenharia química, em diversos momentos há a necessidade de desenvolver modelos, estimar parâmetros e otimizar condições operacionais. Porém, o foco principal está relacionado apenas com a otimização de condições operacionais, em contrapartida, a análise econômica em muitos casos acaba não sendo trabalhada (ARENA et al., 2010). Em virtude disso, a falta de problemas genéricos tratando de assuntos econômicos em reatores químicos deixa os profissionais limitados a dimensionar 0 equipamento (ZAPICO et al., 2015).

Neste contexto, surge a necessidade de uma integração mais efetiva entre a engenharia química e engenharia econômica. $O$ presente trabalho aborda a viabilidade econômica de operação de um reator catalítico de leito fixo (PBR) de uma forma bastante simples. No entanto, tal abordagem pode proporcionar aos estudantes de engenharia química, e também, aos profissionais do meio, uma visão de como a alteração de parâmetros iniciais do processo pode maximizar o lucro de uma indústria.

O objetivo deste trabalho foi analisar o efeito da quantidade de excesso de reagente na entrada de um PBR sobre o lucro com a venda do produto no primeiro mês de operação do reator. A simulação foi utilizada para comparar o lucro obtido de forma paralela ao custo de aquisição de um catalisador de alto valor agregado. Para isto, desenvolveu-se uma modelagem matemática para representar o sistema considerando contração gasosa e perda de carga devido ao escoamento em meio poroso.

\section{MATERIAL E METODOS}

O problema proposto neste trabalho consiste em um sistema de produção de um composto "C", utilizando um reator de leito fixo, PBR, isotérmico. O produto é obtido através da reação elementar $A+2 B \rightarrow C$, em fase gasosa. $A$ mistura prossegue até um condensador, que remove todo composto "C", condensável, permanecendo os compostos "A" e "B" na fase gasosa remanescente, conforme ilustrado na Figura 1.

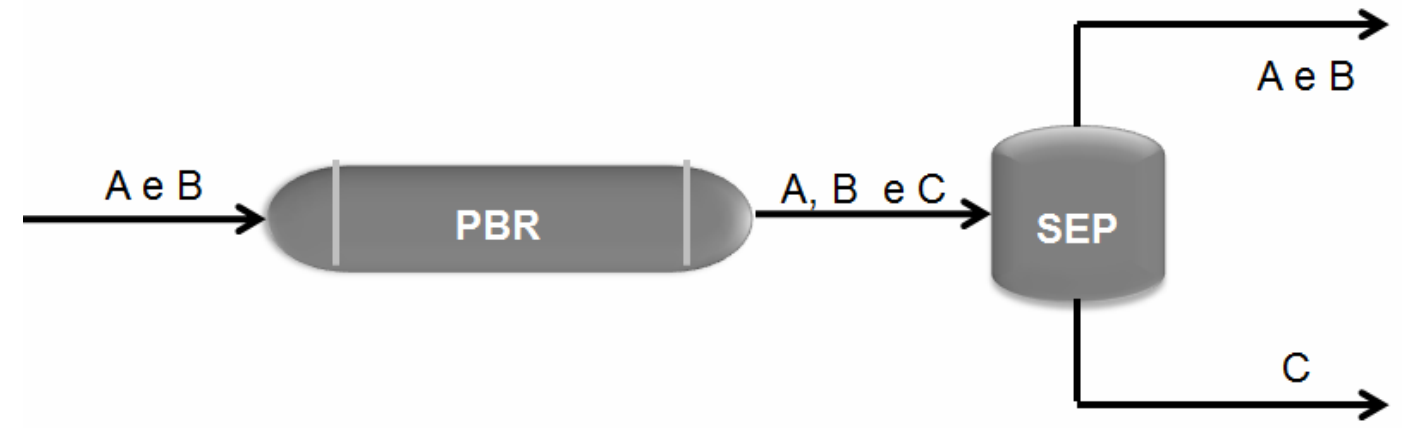

FIGURA 1. Esquema simplificado da unidade de produção do composto C. 
Esta reação somente ocorre na presença de um catalisador específico, de alto custo, que deve ser adquirido. O setor financeiro de uma suposta indústria só aceitará a ativação do reator com a condição de que a produção desse reator cubra os investimentos com catalisador já na primeira receita líquida mensal. Neste primeiro mês, os custos operacionais do reator e do condensador serão cobertos pela produção de outros setores da fábrica.

A questão a ser discutida refere-se à massa de catalisador a ser adquirida para que se obtenha a maior produção de "C" possível, respeitando as restrições do setor financeiro. Para conduzir esta análise, desenvolveu-se uma modelagem matemática para simular o processo, tendo por base a taxa da reação elementar, representada na Equação 1.

$$
r_{A}=-k^{\prime} C_{A} C_{B}^{2}
$$

Uma vez que a reação se processa em fase gasosa, com contração volumétrica e, também, com perda de carga devido ao escoamento no leito poroso, esses termos devem ser incluídos na equação de taxa. Através da estequiometria da reação, representando as concentrações das espécies em função da conversão do reagente limitante "A", a Equação 1 passa a ser representada pela Equação 2.

$$
r_{A}=-k^{\prime}\left[C_{A 0} \frac{\left(1-X_{A}\right)}{\left(1+\varepsilon_{A} X_{A}\right)} \frac{P}{P_{0}}\right] \times\left[C_{A 0} \frac{\left(\Theta_{B}-2 X_{A}\right)}{\left(1+\varepsilon_{A} X_{A}\right)} \frac{P}{P_{0}}\right]^{2}
$$

Em que:

$\mathrm{C}_{\mathrm{A} 0}$ : concentração $(\mathrm{mol} / \mathrm{L})$ do reagente $\mathrm{A}$, limitante;

$\mathrm{X}_{\mathrm{A}}$ : conversão de $\mathrm{A}$;

$\varepsilon_{A}:$ fator de contração volumétrica;

$P:$ pressão total na saída do reator;

$P_{0}$ : pressão total na entrada do reator;

$\theta_{\mathrm{B}}$ : razão entre $\mathrm{C}_{\mathrm{B} 0}$ e $\mathrm{C}_{\mathrm{AO}}$.

Representando a razão $P / P_{0}$ por $y$, e rearranjando a Equação 2, chega-se à Equação 3.

$$
r_{A}=-k^{\prime} C_{A 0}^{3} \frac{\left(1-X_{A}\right)\left(\Theta_{B}-2 X_{A}\right)^{2}}{\left(1+\varepsilon_{A} X_{A}\right)^{3}} y^{3}
$$

O balanço molar simplificado e ajustado para a conversão de $A$, em regime permanente, no PBR está representado através da Equação 4. Enquanto que a perda de carga no leito de catalisador foi representada pela Equação 5, que referese à uma adaptação da equação de Ergun.

$$
\frac{d X_{A}}{d W}=\frac{k^{\prime} C_{A 0}^{3}}{F_{A 0}} \frac{\left(1-X_{A}\right)\left(\Theta_{B}-2 X_{A}\right)^{2}}{\left(1+\varepsilon_{A} X_{A}\right)^{3}} y^{3}
$$


Sendo,

W: massa de catalisador;

$\mathrm{F}_{\mathrm{A} 0}$ : vazão molar de $A$ na entrada do reator.

$$
\frac{d y}{d W}=-\frac{\alpha}{2 y}\left(1+\varepsilon_{A} X_{A}\right)
$$

Em que:

a: parâmetro de perda de carga;

$\mathrm{y}$ : razão entre $\mathrm{P}$ e $\mathrm{P}_{0}$.

As Equações (4) e (5) foram resolvidas numericamente, de forma simultânea, utilizando o método de Euler em planilha do Excel® para uma massa de catalisador variando de 0 a $30 \mathrm{~kg}$, com passos de integração de $0,01 \mathrm{~kg}$. As condições iniciais do problema foram: para $W=0, X_{A}=0$ e para $W=0, y=1$. Após a obtenção dos perfis de conversão, as vazões molares de cada componente foram determinadas através da estequiometria da reação. O Quadro 1 fornece o conjunto de parâmetros utilizados nas simulações do problema.

QUADRO 1. Conjunto de parâmetros utilizados nas simulações.

\begin{tabular}{ll}
\hline Parâmetro & valor \\
\hline $\mathrm{C}_{\mathrm{A} 0}$ & $0,1 \mathrm{kmol} / \mathrm{m}^{3}$ \\
$\mathrm{C}_{\mathrm{B} 0}$ & $0,2 \mathrm{e} 0,4 \mathrm{kmol} / \mathrm{m}^{3}$ \\
$\mathrm{k}^{\prime}$ a $350 \mathrm{~K}$ & $12 \mathrm{~m}^{9} \mathrm{kmol}^{-2} \mathrm{kgcat}^{-1} \mathrm{~h}^{-1}$ \\
Vazão total de entrada, $\mathrm{v}_{0}$ & $7 \mathrm{~m}^{3} / \mathrm{h}$ \\
$\alpha$ & $0,037 \mathrm{~kg}^{-1}$ \\
$\mathrm{M}_{\mathrm{C}}$ & $300 \mathrm{~kg} / \mathrm{kmol}$ \\
Valor de venda de C & $0,64 \mathrm{R} \$ / \mathrm{kmol}$ \\
Custo do catalisador & $4000 / \mathrm{kg}$ \\
$\varepsilon_{\mathrm{A}}$ & $-2 / 3$ \\
\hline
\end{tabular}

A relação entre os custos e receitas foi obtida através da análise dos pontos de coincidência da Figura 4. Este gráfico fornece o custo em função da massa de catalisador; e o valor, em reais, das receitas referentes à comercialização do produto $C$ para uma determinada massa de catalisador. Utilizaram-se dois valores de $C_{B 0} e$, para cada um, verificou-se a produtividade de C. Com isto, cada receita pode ser comparada com o custo de aquisição do catalisador.

\section{RESULTADOS E DISCUSSÃO}

A Figura 2 fornece uma análise da influência da concentração de reagente $B$ sobre a conversão do reagente limitante em função da massa de catalisador. Avaliaram-se concentrações de $B$ iguais a 0,2 $M$ e 0,4 M. Verificou-se que o aumento na concentração de B promove um aumento na conversão de A para uma mesma massa de catalisador. Este resultado era esperado, visto que a taxa da reação é proporcional a concentração dos reagentes e é influenciada pela concentração com uma ordem de reação igual a 2 . Isso sugere que tal reagente pode ser a chave para obter condições ótimas de operação do sistema.

Ainda em relação à Figura 2 , verifica-se que há uma redução da perda de carga quando a concentração de $\mathrm{B}$ passa de $0,2 \mathrm{M}$ para $0,4 \mathrm{M}$. Este resultado está intimamente relacionado com a conversão de $A$, que aumenta para uma mesma 
massa de catalisador (MAT NOOR et al., 2010; VIEIRA et al., 2015).

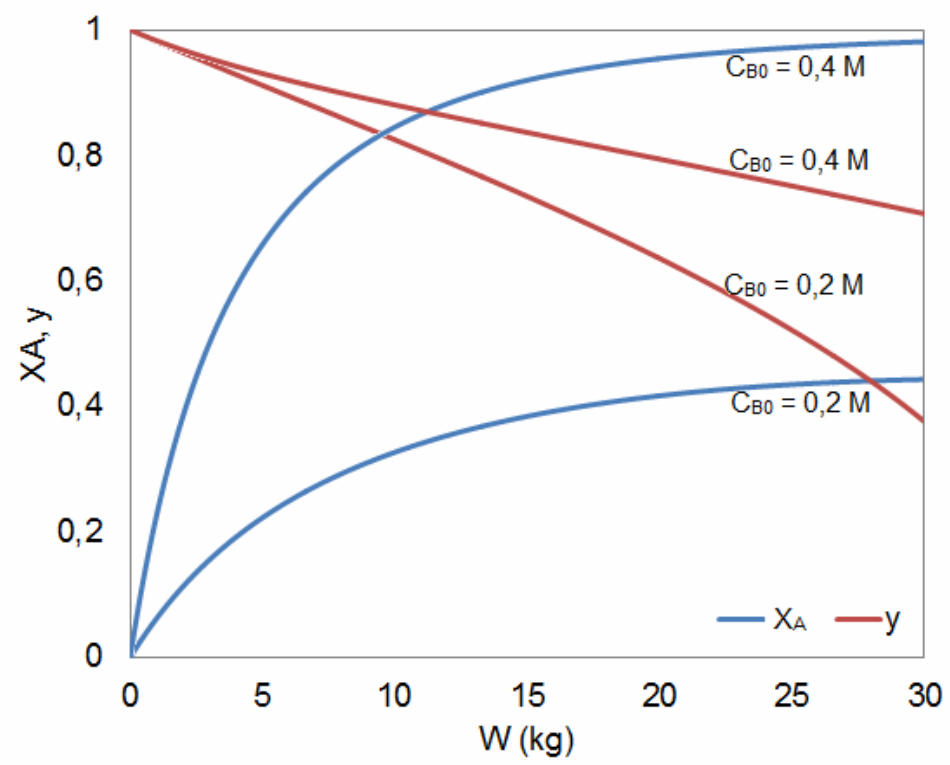

FIGURA 2. Conversão de reagente limitante (linha azul) e perda de carga (linha vermelha) em função da massa de catalisador para $\mathrm{C}_{\mathrm{B} 0}=0,2 \mathrm{M}$ e $\mathrm{C}_{\mathrm{B} 0}=0,4 \mathrm{M}$.

A Figura 3 fornece as vazões molares de cada componente da reação em função da massa de catalisador contida no reator tubular. Através dos valores obtidos nessa figura é possível compreender a magnitude da redução do componente A quando a concentração de B é dobrada. De fato, este resultado complementa o resultado obtido na Figura 2, em que se consegue aumentar a produção de C. Uma vez que o componente C é facilmente condensável, o componente B remanescente poderia ser reciclado para o início do processo, fazendo com que altas concentrações fossem mantidas na entrada.

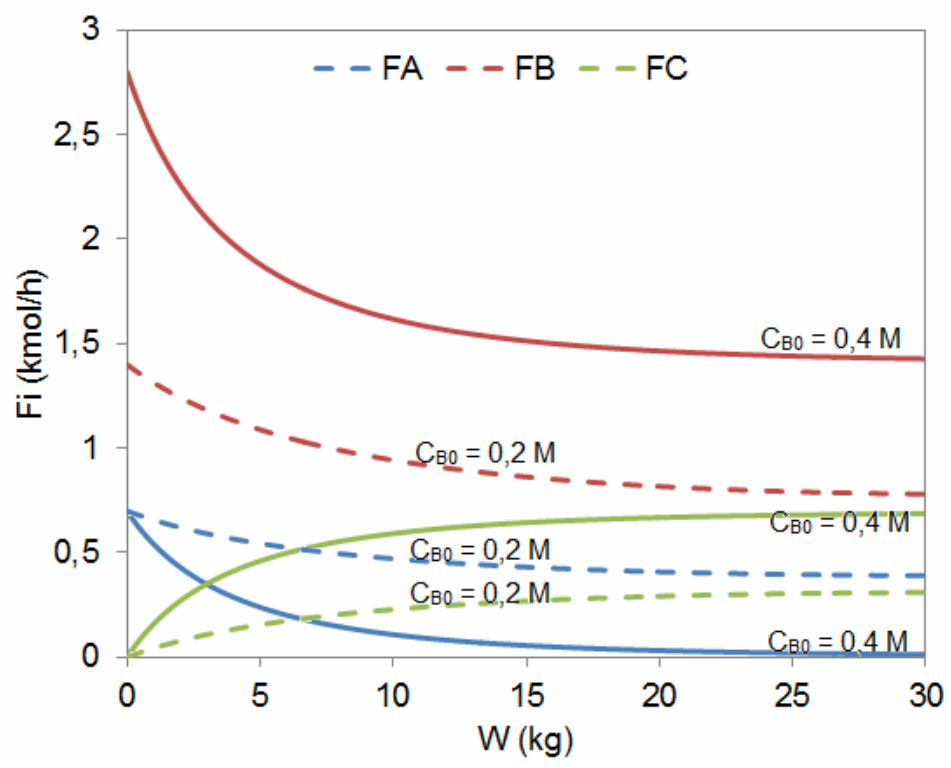

FIGURA 3. Vazões molares de todos os componentes na saída do reator, sendo que as linhas tracejadas representam as vazões para $C_{B 0}=0,2 \mathrm{M}$ e as linhas contínuas representam as vazões para $\mathrm{C}_{\mathrm{B} 0}=0,4 \mathrm{M}$. 
Dispondo-se das vazões molares de cada componente e da massa molar do componente $\mathrm{C}$, construiu-se o gráfico da Figura 4, que ilustra o custo de catalisador (linha reta em azul) e o faturamento com a produção de $C$ (linhas exponenciais em vermelho) no primeiro mês de operação do sistema, ambos em função da massa de catalisador. A análise deste cenário é, sem dúvidas, um ponto de partida para se discutir a viabilidade econômica de um reator deste tipo.

Verificando-se a Figura 4, para a concentração de 0,2 $\mathrm{M}$ do componente $\mathrm{B}$ na entrada do reator, nota-se que até uma massa de aproximadamente $7 \mathrm{~kg}$ de catalisador, qualquer produção de $\mathrm{C}$ resulta em uma receita maior do que o custo do catalisador utilizado. Ou seja, a utilização de um PBR com massa de catalisador menor do que este valor pode proporcionar um lucro já no primeiro mês (sem contar os outros custos do processo).

Para reatores com massa de catalisador maior do que este valor será impossível obter lucro no primeiro mês, o que, de fato, dificilmente ocorre em processos industriais. Por outro lado, se for realizada uma análise da maior massa de catalisador utilizada $(30 \mathrm{~kg})$, verifica-se que a receita com as vendas de $C$ no mês ficarão em torno de $R \$ 40.000,00$, e o custo de catalisador em torno de $R \$$ 120.000,00. Deste modo, a recuperação do valor investido em catalisador se dará a partir do terceiro mês de operação.

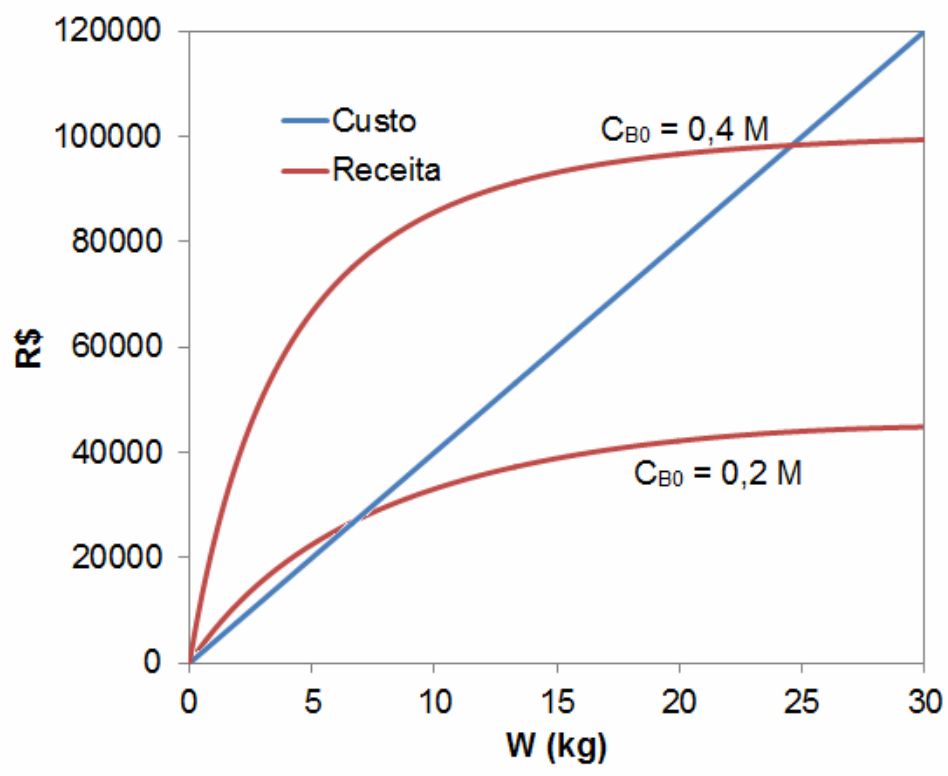

FIGURA 4. Relação entre custo de catalisador e faturamento do mês com a venda do produto $\mathrm{C}$.

Ainda referindo-se à Figura 4, para a concentração de $B$ na entrada do reator igual a 0,4 M nota-se uma grande produção de $C$ para pequenas massas de catalisador utilizadas. Esta produção gerou valores de faturamento bem maiores do que o custo de catalisador para massas de até $23 \mathrm{~kg}$ (em que há um ponto de equilíbrio). Ou seja, utilizar o reagente $\mathrm{B}$ em excesso proporciona vantagens econômicas que podem ter um impacto considerável no lucro final da indústria. Por exemplo, se for utilizado um PBR com $10 \mathrm{~kg}$ de catalisador, com custo de $R \$$ $40.000,00$, haverá uma produção de $C$ gerando uma receita de aproximadamente $\mathrm{R} \$ 86.000,00$, que é mais do que o dobro investido em catalisador. Vale ressaltar que este resultado é referente apenas ao primeiro mês de operação do sistema. 


\section{CONCLUSÃO}

O objetivo deste trabalho foi mostrar aos leitores como o excesso de um determinado reagente pode impactar positivamente no faturamento da produção de um composto genérico em um reator catalítico de leito fixo. Verificou-se que, para o sistema em questão, utilizar excesso de "B" promove um aumento da taxa de reação e, com isto, maior conversão do reagente limitante para uma mesma massa de catalisador. Por exemplo, obteve-se um lucro de $115 \%$ em relação à massa de catalisador adquirida $\left(10 \mathrm{~kg}\right.$ ) para $\mathrm{C}_{\mathrm{B} 0}=0,4 \mathrm{M}$; enquanto que para $\mathrm{C}_{\mathrm{B} 0}=0,2 \mathrm{M}$ houve um prejuízo de $17 \%$ para a mesma massa de catalisador utilizada no primeiro mês. Através de uma análise mais detalhada utilizando várias concentrações de $\mathrm{B}$ e informações sobre o custo de reagentes e outros equipamentos, um valor ótimo para a operação do sistema poderia ser obtido. Sendo, portanto, este trabalho um ponto de partida para a análise de viabilidade econômica de sistemas reacionais análogos.

\section{AGRADECIMENTOS}

Os autores deste trabalho agradecem ao Instituto Federal de Educação, Ciência e Tecnologia do Sul de Minas Gerais - IFSULDEMINAS, pela colaboração financeira.

\section{REFERÊNCIAS}

ARENA, F.; ITALIANO, C.; RANERI, A.; SAJA, C. Mechanistic and kinetic insights into the wet air oxidation of phenol with oxygen (CWAO) by homogeneous and heterogeneous transition-metal catalysts. Applied Catalysis B: Environmental, v. 99, n. 1-2, p. 321-328, 2010. Disponível em: <http://linkinghub.elsevier.com/retrieve/pii/S0926337310002894>. doi: doi.org/10.1016/j.apcatb.2010.06.039.

CHACHUAT, B.; LATIFI, M. A. A new approach in deterministic global optimisation of problems with ordinary differential equations. Frontiers In Global Optimization, v. 74, p. 83-108, 2003. Disponível em: < https://link.springer.com/chapter/10.1007/9781-4613-0251-3_5 >. doi: 0.1007/978-1-4613-0251-3_5.

MAT NOOR, R. A.; AHMAD, Z.; MAT DON, M.; UZIR, M. H. Modelling and control of different types of polymerization processes using neural networks technique: $A$ review. The Canadian Journal of Chemical Engineering, v. 88, n. 6, p. 1065-1084, 2010. Disponível em: <http://doi.wiley.com/10.1002/cjce.20364>. doi: 10.1002/cjce.20364.

SILVA, N. L.; CRISPIM, J. M. S.; VIEIRA, R. P. Kinetic and Thermodynamic Analysis of Anthocyanin Thermal Degradation in Acerola (Malpighia emarginata D.C.) Pulp. Journal of Food Processing and Preservation, 2016 (In press). Disponível em: $<$ http://doi.wiley.com/10.1111/jppp.13053>. doi: 10.1111/jppp.13053.

VIEIRA, R. P.; LONA, L. M. F. Optimization of reaction conditions in functionalized polystyrene synthesis via ATRP by simulations and factorial design. Polymer Bulletin, v. 73, n. 7, p. 1795-1810, 2016. Disponível em: <http://link.springer.com/10.1007/s00289-015-1577-z>. doi: 10.1007/s00289-0151577-z. 
VIEIRA, R. P.; LONA, L. M. F. Kinetic modeling of atom-transfer radical polymerization: inclusion of break reactions in the mechanism. Polymer Bulletin, $v$. 73, n. $\quad 8, \quad$ p. 2105-2119, 2016. Disponível em: <http://link.springer.com/10.1007/s00289-015-1596-9>. doi: 10.1007/s00289-0151596-9.

VIEIRA, R. P.; OSSIG, A.; PEREZ, J. M.; GRASSI, V. G.; PETZHOLD, C. L.; PERES, A. C.; COSTA, J. M.; LONA, L. M. F. Styrene ATRP Using the New Initiator 2,2,2-Tribromoethanol: Experimental and Simulation Approach. Polymer Engineering and Science, v. 55, p. 2270-2276, 2015. Disponível em: <http://onlinelibrary.wiley.com/doi/10.1002/pen.24113/abstract>. doi: 10.1002/pen.24113.

ZAPICO, R. R.; MARÍN, P.; DÍEZ, F. V; ORDÓÑES, S. Influence of operation conditions on the copper-catalysed homogeneous wet oxidation of phenol: development of a kinetic model. Chemical Engineering Journal, v. 270, p. 122132, $2015 . \quad$ Disponível em: http://www.sciencedirect.com/science/article/pii/S1385894715001497>. doi: doi.org/10.1016/j.cej.2015.01.112. 